\title{
A Novel Location-aided Routing Algorithm for Mobile Ad Hoc Network with a Small Expenditure on Route-Discovery
}

\author{
Hong Peng*, Shi-yuan Fu, Li-min Meng \\ College of Information Engineering, Zhejiang University of Technology, \\ Zhejiang Provincial Key Laboratory of Communication Networks and Applications, \\ Hangzhou, 310023, China \\ Xia Liu \\ EME Group, Kordia ${ }^{\mathrm{TM}}$ Solutions, 11 Ashtan Place, \\ Banyo, Queensland, 4014, Australia \\ Received 21 October 2011 \\ Accepted 15 June 2012
}

\begin{abstract}
The location-aided routing algorithm adopts the flooding mechanism to transmit route control packet in mobile Ad Hoc networks. Such mechanism not only affects nodes which do not join the final route, but also needs a large number of unnecessary deliveries of control packets. To address this issue, we propose a novel location-aided routing algorithm based on Grover quantum search theory in this paper. Simulation results show that our proposed algorithm can reduce the number of relative nodes and the expenditure on route-discovery.
\end{abstract}

Keywords: Grover search algorithm, Mobile Ad Hoc Network, Location-aided routing, expenditure on routediscovery.

\section{Introduction}

Mobile Ad Hoc Network (MANET) is an emerging wireless communication network which consists of a group of mobile nodes with wireless transceivers. Applications of MANET have been growing rapidly in the past decades, which include military communications, emergency services, vehicular communications and so on. ${ }^{1}$

Due to the lack of infrastructure, MANET usually requires distributed routing algorithms for routing. Therefore, every node in MANET has to collaborate on route-discovery. However, due to the mobility and multi-hop, it is generally difficult to search for an efficient route with the low computational complexity in MANET. Therefore, it is important to develop a real- time routing algorithm for MANET which lowers the expenditure on route-discovery and fastens the convergence speed.

Recently, various routing algorithms have been proposed for MANET in the existing literatures. Some of the routing algorithms reduce expenditure on routediscovery through drawing upon needed the assistance of location information, e.g., Location-Aided Routing (LAR) algorithm, ${ }^{2}$ Distance Routing Effect Algorithm for Mobility (DREAM) algorithm, ${ }^{3}$ Greedy Perimeter Stateless Routing (GPSR) algorithm ${ }^{4}$ and so on. Although these existing algorithms have their own advantages, they still suffered from some drawbacks. For instance, the flooding mechanism is used in the LAR algorithm to blindly transmit the control packets for route-discovery in the request-zone, which results in

* Corresponding author: ph@zjut.edu.cn 
a large number of unnecessary packet-deliveries. This situation may become even worse if the density of mobile nodes in the request-zone increases. ${ }^{5}$ Therefore, it is of importance to develop a new routing algorithm to reduce the overhead due to the blind flooding mechanism.

As an intelligent algorithm, Grover search algorithm ${ }^{6}$ aims at solving unstructured search problems by using the special characteristics of wave. Because of the huge storage capacity, Grover search algorithm can perform unstructured search with a high-speed parallel computation. In particular, Grover search algorithm not only converges to the solution-set quickly (in a probabilistic measure), but also strikes a balance between the complexity and the robustness of iterations. Thanks to these properties, we propose a routing algorithm, referred to as Grover Location-Aided Routing (G-LAR) algorithm, based on the idea of Grover search algorithm for the route-discovery in MANET. In the G-LAR algorithm, the intermediate nodes only choose the nodes with higher transmit probability as next-hops. Therefore, it can efficiently increase the probability of transmitting control packets for nodes included in the final selected route. On the contrary, the probability of transmitting control packets is reduced for nodes excluded in the final selected route. Furthermore, the number of relative nodes and the overhead due to unnecessary deliveries of control packets are reduced in route-discovery.

\section{A Brief Review of the LAR Algorithm}

Before introducing our proposed G-LAR algorithm, we first review the LAR algorithm in this section.

The LAR algorithm is proposed based on the classical Dynamic Source Routing (DSR) algorithm, ${ }^{7}$ and thus it belongs to the category of the on-demand routing algorithms based on source-routing. In the LAR algorithm, a special zone for route-discovery is defined according to the nodes' real-time geographic positions, which can be obtained from the Global Position System (GPS). In particular, the LAR algorithm only invokes the operations of the nodes within this special zone for route-discovery, thus reducing the operational complexity compared to the DSR algorithm relying on the flooding of all the nodes.
Let $\left(x_{d}\left(t_{0}\right), y_{d}\left(t_{0}\right)\right)$ denote the position of the destination node $\mathrm{D}$ at time $t_{0}$ and let $v_{d}(t)$ denote its moving speed at time $t$. Therefore, the zone in which node $\mathrm{D}$ will appear at time $t_{1}$ is given by a circular region as follows:

$$
0<\sqrt{\left(x_{d}\left(t_{0}\right)-x(t)\right)^{2}+\left(y_{d}\left(t_{0}\right)-y(t)\right)^{2}}<R,
$$

where $R=v_{d}(t) \cdot\left(t_{1}-t_{0}\right)$. We call this circular region as the expected-zone in the rest of this paper. In LAR, the request-zone for route-discovery is the smallest rectangle defined by the source node and the expectedzone. In particular, the request-zone is set according to the following two conditions. ${ }^{8}$ The detailed illustrations are shown in Fig.1 and Fig.2.

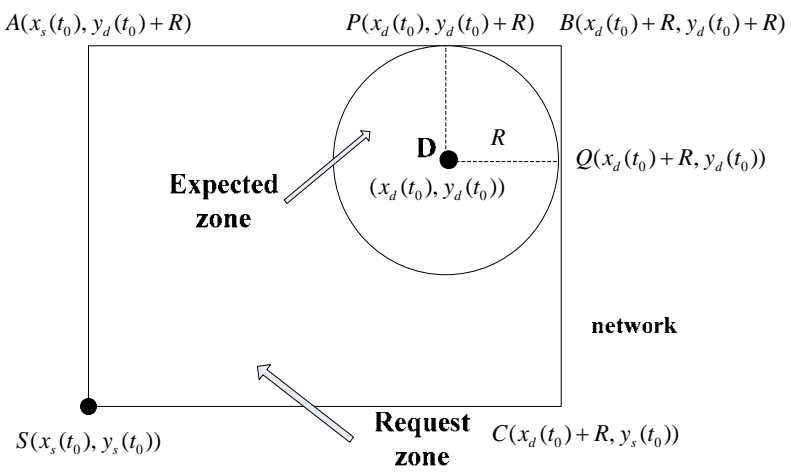

Fig. 1. Source node outside the expected zone

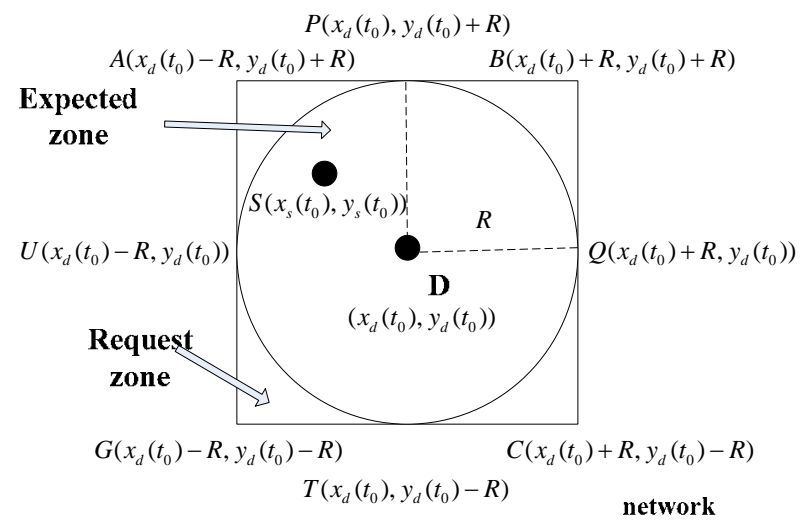

Fig. 2. Source node inside the expected zone

Condition 1: the source node is outside the expected zone, which is mathematically given by Eq. (2). 


$$
\left\{\begin{array}{l}
x_{s}\left(t_{0}\right) \leq x(t) \leq x_{d}\left(t_{0}\right)+R \\
y_{s}\left(t_{0}\right) \leq y(t) \leq y_{d}\left(t_{0}\right)+R
\end{array}\right.
$$

Condition 2: the source node is inside the expected zone, which is mathematically given by Eq. (3).

$$
\left\{\begin{array}{l}
x_{d}\left(t_{0}\right)-R \leq x(t) \leq x_{d}\left(t_{0}\right)+R \\
y_{d}\left(t_{0}\right)-R \leq y(t) \leq y_{d}\left(t_{0}\right)+R
\end{array}\right.
$$

Similar to the DSR algorithm, the LAR algorithm uses the flooding mechanism to transmit packets for the route-discovery. However, in the LAR algorithm, only the nodes in the request-zone participate in the route discovery. The nodes outside the request-zone will drop the received route control packets. The request-zone is predetermined by the source node $S$ in the route requestpacket. When an intermediate node receives this route request-packet, it will check whether it belongs to this specified request-zone or not. If it is true, then the intermediate node will flood the request-packet. Otherwise, it will drop the received request-packet. If the source node $\mathrm{S}$ does not receive any reply of the route packet within a certain interval, implying a failure of route-discovery, then the source node $\mathrm{S}$ will enlarge the request-zone and carry out the route discovery procedure once again. The extreme case is that the request-zone becomes the entire network.

It is apparent that the area of the request-zone depends on the node's moving speed $v_{d}(t)$ and the time $\left(t_{1}-t_{0}\right)$ needed to update the position information. If the node moves quickly or the time of updating the position information is long, then the request-zone will be large. A large request-zone with high node density has nodes to exchange route control packets frequently, which results in a large number of congestions and high overhead on route-discovery.

\section{Grover Search Algorithm}

We develop a novel location-aided routing algorithm based on the idea of Grover search algorithm. Therefore, the idea of Grover search algorithm is briefly introduced before we present our algorithm.

Grover search algorithm has a huge storage capacity and is able to perform unstructured search with a highspeed parallel computation. ${ }^{9}$ Therefore, unlike classical search algorithms which find a target within a set of
$N$ nodes with the complexity of $\mathrm{O}(N)$, Grover search algorithm $^{6}$ (proposed by Grover in 1996) has the complexity of $\mathrm{O}(\sqrt{N})$. In particular, Fig. 3 shows the skeleton of the Grover search algorithm, which includes the following three steps.

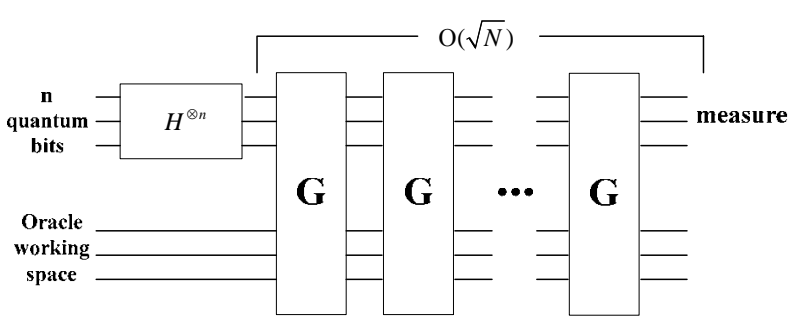

Fig. 3. Skeleton of Grover search algorithm

(Step i) Initialization. The Grover search algorithm uses the quantum-bit as its basic unit for calculation. Specifically, $n$ quantum-registers can storage $N=2^{n}$ quantum ground states, representing $N$ unordered nodes in the network. Nodes in MANET are unsorted with equal probability to appear, so the initial state of network is given by Ref. 10 as follows:

$$
|\varphi\rangle=\frac{1}{\sqrt{N}} \sum_{x=0}^{N-1}|x\rangle .
$$

(Step ii) Grover Iteration. Starting from the above initial state, we perform Grover iteration repeatedly to update the probability of each quantum ground state. The objective is to maximize the probability of the quantum ground state which meets the criterions for searching.

(Step iii) Comparison and Output. Grover iteration outputs the quantum ground state with the largest probability.

Figure 4 shows the procedure of Grover iteration (i.e., the above Step ii). Due to the space limitation, we omit the details here. The interested readers are referred to Ref. 11 for the details on Grover iteration.

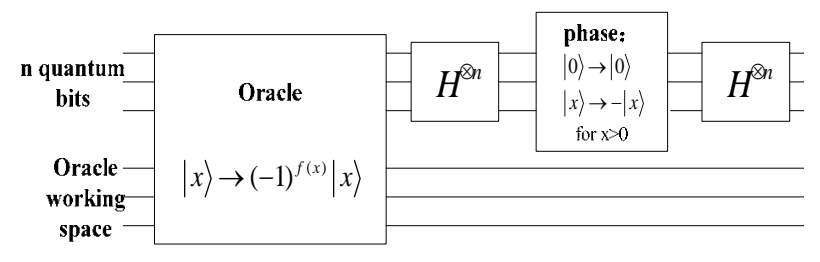

Fig. 4. Procedure of Grover iteration 
Geometrically, Grover iteration can be considered as the rotation from the initial state to a "desirable" state. In order to explain Grover iteration clearly, let $|\alpha\rangle$ and $|\beta\rangle$ denote two different quantum ground states. $|\beta\rangle=\sum_{x}|x\rangle / \sqrt{M}$ are the states of searching targets of all $x .|\alpha\rangle=\sum_{x} "|x\rangle / \sqrt{N-M}$ are the rest of the states. $M$ denotes the number of searching targets. Since only a single route will be selected in the route-discovery, i.e. $M=1$, the initial state of the network can be given by Eq. (5).

$$
|\varphi\rangle=\sqrt{\frac{N-1}{N}}|\alpha\rangle+\sqrt{\frac{1}{N}}|\beta\rangle
$$

In each round of Grover iteration, the initial state will be rotated by an angle of $\theta\left(0<\theta<\frac{\pi}{2}\right)$, where $\theta=\cos ^{-1} \sqrt{\frac{N-1}{N}}$. After $k$-th round of the Grover iteration, the initial state will be changed to Eq. (6) according to Ref. 12.

$$
G^{k}|\varphi\rangle=\cos \left(\frac{2 k+1}{2} \theta\right)|\alpha\rangle+\sin \left(\frac{2 k+1}{2} \theta\right)|\beta\rangle
$$

Figure 5 illustrates the Grover iteration in a single round of iteration.

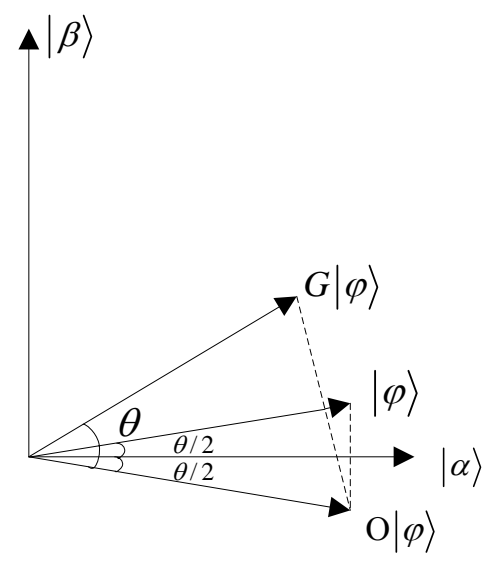

Fig. 5. Geometric view of Grover iteration in a single round

When the initial state vector approaches to $|\beta\rangle$ after several rounds of Grover iteration, we can measure the exact probability of the searching targets. According to Ref.13, the earliest time to reach the near-optimal measurement is $\sqrt{N}$, thus our following proposed algorithm performs $\sqrt{N}$ rounds of Grover iterations.

\section{Proposed G-LAR Algorithm}

In this section, we propose the G-LAR algorithm based on the idea of Grover search algorithm for routediscovery.

The G-LAR algorithm requires three important matrixes (namely, the link matrix, the operating matrix and the span matrix) which reflect the conditions of networks. ${ }^{14}$

First, we construct the link matrix to reflect the connectivity of the nodes within the request-zone. With the assistance of GPS, we know node $i$ 's position $\left(x_{i}(t), y_{i}(t)\right)$, its moving speed $v_{i}(t)$ and the moving direction $\theta_{i}(t)$ at time $t$. Based on such information, the distance between two nodes can be calculated. If the distance between two nodes is shorter than the maximum range for wireless communication, these two nodes can exchange packets directly. Otherwise, their communication needs the relay of other nodes. We construct a $N \times N$ link matrix $A(t)=\left(a_{i j}(t)\right)_{N \times N}$ to show the connectivity of the network with $N$ mobile nodes. Specifically, each element of the link matrix is given by

$$
a_{i j}(t)=\left\{\begin{array}{ll}
1 & 0 \leq l_{i j}(t) \leq r \\
0 & \text { others }
\end{array},\right.
$$

where $l_{i j}(t)$ is the real-time distance between node $i$ and node $j$ at time $t$, and $r$ is the maximum range for wireless communication. $a_{i j}(t)=1$ means that node $i$ and node $j$ can communicate with each other directly, and $a_{i j}(t)=0$ means the opposite case.

Second, we define an $N \times N$ operating matrix $U^{(i)}(t)=\left(u_{m n}^{(i)}(t)\right)_{N \times N}$ for the nodes within the request-zone as follows:

$$
u_{m n}^{(i)}(t)=\left\{\begin{array}{ll}
1 & m=n \& \& a_{m i}(t)=0 \\
-1 & m=n \& \& a_{m i}(t)=1 . \\
0 & m \neq n
\end{array} .\right.
$$

Specifically, $u_{m n}^{(i)}(t)=-1$ means that the local node $i$ and node $m$ are neighbors, and they can communicate 
with each other directly (and hence node $m$ could be a feasible next-hop node). To further avoid loop phenomenon, we set node $i$ and its previous-hop node $j$ to $u_{j j}^{(i)}(t)=1$.

Third, we construct the span matrix $D^{(i)}(t)=\left(d_{i j}^{(i)}(t)\right)_{N \times N}$ for Grover iteration. The span matrix is a unitary matrix with the objective to enlarge the target probability quickly. In our proposed G-LAR algorithm, the criterion of the minimum hops yields the span matrix as follows:

$$
d_{i j}^{(i)}(t)=\left\{\begin{array}{ll}
\frac{2}{N} & j \neq i \\
\frac{2}{N}-1 & j=i
\end{array} .\right.
$$

At the beginning of the route discovery, a $1 \times N$ probability vector $\alpha$ will be fixed in the route request packet to record the transmit probability of nodes for their next-hops. The initial probability is $1 / \sqrt{N}$ based on Eq. (4). When a local node $i$ receives the route request packet in the request-zone, it first constructs the three matrixes aforementioned. Then, it picks up the probability $\alpha_{k}$ at the previous hop and calculates the new transmit probability $\alpha_{k+1}$ as follows:

$$
\alpha_{k+1}=\left(U^{(i)} D\right)^{[\sqrt{N}]} \alpha_{k}
$$

Figure 6 is the flow chart of G-LAR algorithm. In the G-LAR algorithm, when the density of the nodes is small, we use the flooding mechanism to transmit the route control packets in request-zone to avoid unnecessary attempts of route discovery. However, when the number of nodes is more than $M$, we use Grover search algorithm to calculate the transmit probability to reduce nodes to be influenced. We will choose nodes with probabilities above a specified value $U$ to be the next-hops. In other words, instead of flooding packets to every neighbor nodes blindly, we only choose the nodes which are highly likely to be part of the finally selected route to transmit. Sufficient numerical results indicate that our G-LAR algorithm performs better when the values of $M, U$ are chosen to be 8 and 0.5 , respectively.

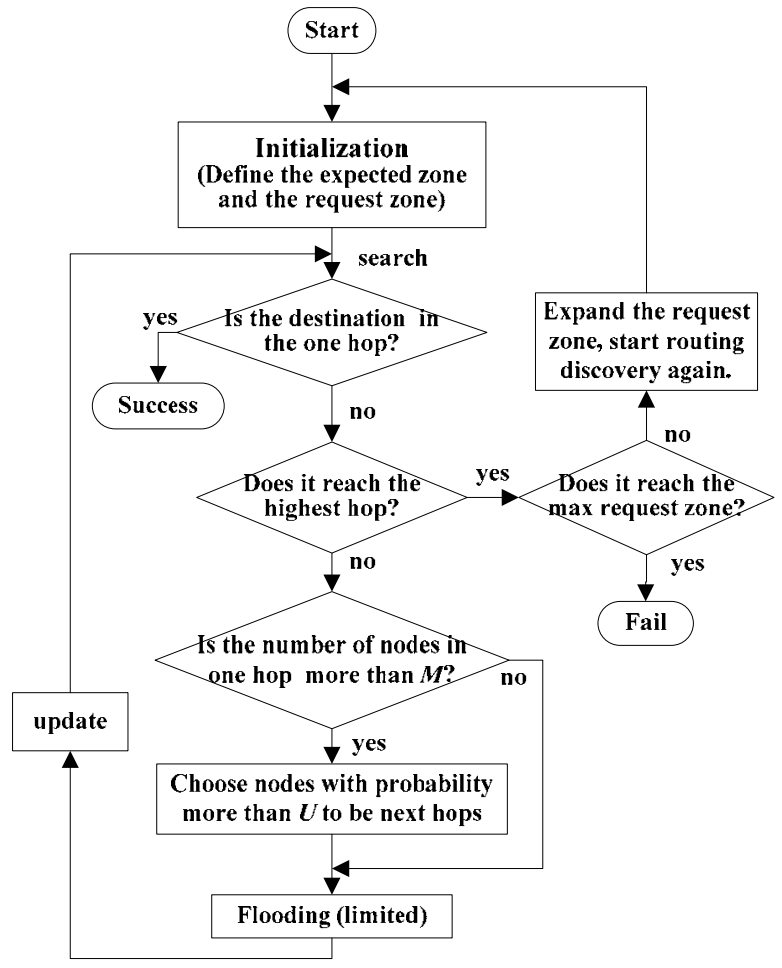

Fig. 6. The flow chart of the proposed G-LAR routing algorithm

\section{Simulation Results}

In this section, we evaluate the performance of our proposed G-LAR algorithm through sufficient numerical experiments built on the OPNET Modeler, ${ }^{15}$ a widely used tool for network simulation. Specifically, we build a MANET consisting of 50 nodes within an area of $1000 \mathrm{~m} \times 1000 \mathrm{~m}$. Each node in the MANET has two states, i.e., the stationary state and the mobile state (with the moving-speed $2 \mathrm{~m} / \mathrm{s}$ ). The IEEE 802.11 protocol is adopted in the nodes' physical layer and MAC layer, and the maximum range for wireless communication is 300 meters.

We consider two different simulation scenarios of mobile and stationary nodes, respectively. In the mobile scenario, nodes can move, hence the topology is timevarying. However, in the stationary scenario, nodes are fixed, thus forming a static topology. Each scenario lasts for 2400 seconds with 1280 seeds. The whole 2400 seconds are cut into 80 intervals with each interval lasts for 30 seconds. 
The main part of the whole traffic sent into the MANET is the routing traffic, including that for the route discovery and that for the route maintenance. The routing traffic is a direct measure to compare the performance of routing algorithm. Specifically, the smaller the routing traffic, the better the performance of the routing algorithm in terms of additional overhead expenditure. Figures 7 and 8 show our proposed G-LAR algorithm achieves average decreases in the routing traffic by $5 \%$ (in stationary scenario) and $10 \%$ (in mobile scenario) compared to the conventional LAR algorithm, respectively. The benefit of our G-LAR algorithm stems from that it selectively chooses the neighbor nodes to send the route control packets, instead of blindly flooding the packets to all the neighbor nodes as the conventional LAR algorithm. Therefore, the number of nodes influenced in the route discovery is reduced, so does the corresponding routing traffic.

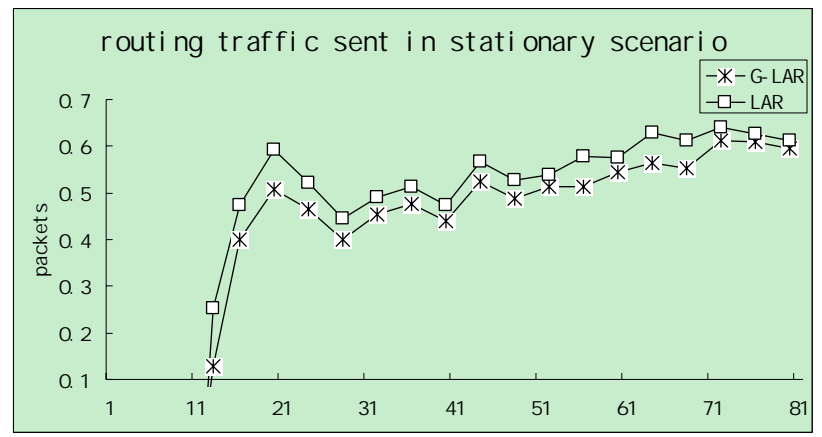

Fig. 7. Routing traffic sent in stationary scenario

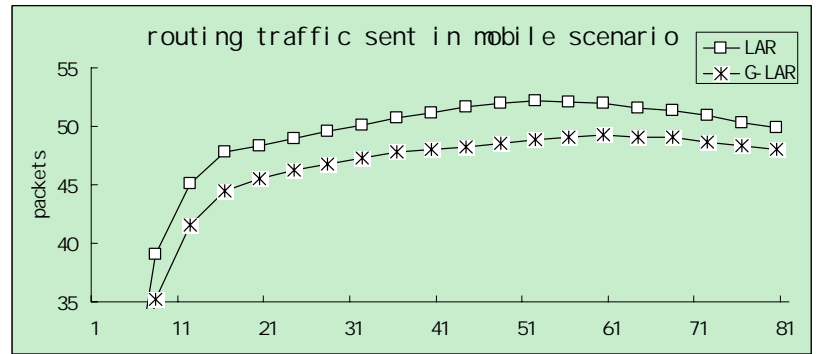

Fig. 8. Routing traffic sent in mobile scenario

The traffic for acknowledgement, including those for the acknowledgement of packets salvaging, those for the acknowledgement to confirm the link condition between nodes and so on, is another important part of the expenditure in the route discovery. Specifically, the comparison between Fig. 9 (with a stationary topology) and Fig. 10 (with a dynamic topology) shows that the change of topology will cause the increase of the acknowledgement dramatically. The acknowledgement is less than 25 packets in the stationary scenario. However, it rises up to more than 1500 packets in the mobile scenario. Since the mobility of the nodes incurs the changes of link conditions frequently, each node has to send the acknowledgement frequently to check whether it can communicate with its neighborhoods directly. Small amount of aggregate acknowledge definitely is preferred for MANET since it will leads to less traffic jamming and small delay. Our proposed GLAR algorithm achieves this desired property. Specifically, Fig. 9 and Fig. 10 show that our G-LAR algorithm reduces the acknowledgements by $15 \%$ in the stationary scenario and $8 \%$ in the mobile scenario, respectively, compared to the conventional LAR algorithm.

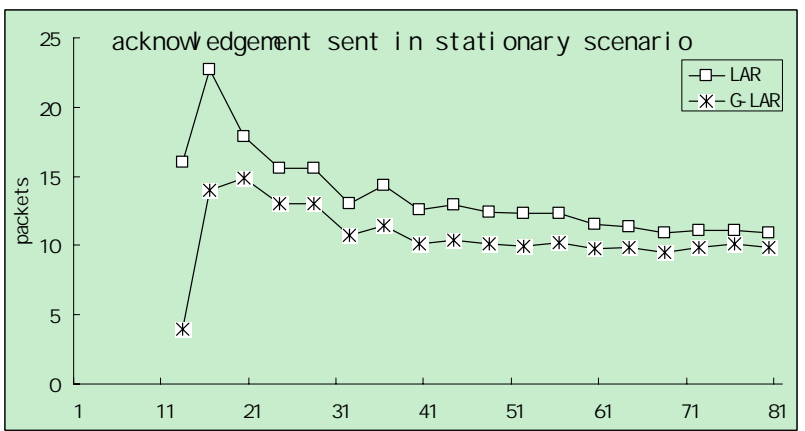

Fig. 9. Acknowledgement sent in stationary scenario

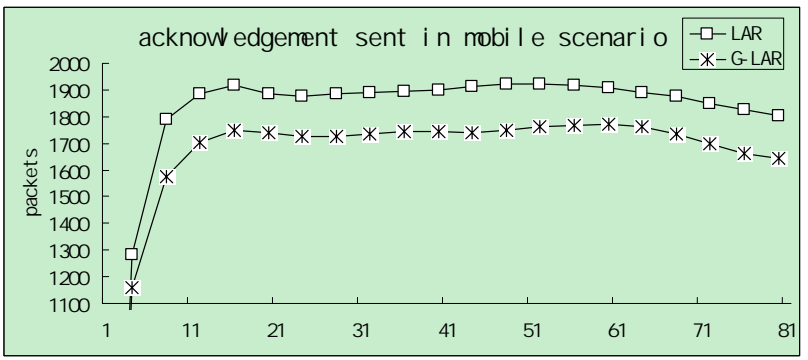

Fig. 10. Acknowledgement sent in mobile scenario

The environment of MANET is complicated in practice, with a lot of factors influencing the successful delivery of the packets. For instance, the changing 
topology will make the routing information out-of-date quickly and lead to packet-loss consequently. In the LAR algorithm, all nodes in the request-zone are influenced in the route discovery with more packets delivered than our proposed G-LAR algorithm. Therefore, the packets-loss happens more frequently when the LAR algorithm is used. Figures 11 and 12 verify this point. Specifically, Fig. 12 indicates that the packets loss rate increases in the mobile scenario due to the dynamic topology. However, our proposed G-LAR outperforms the conventional LAR algorithm by $18 \%$ in terms of the packets loss rate.

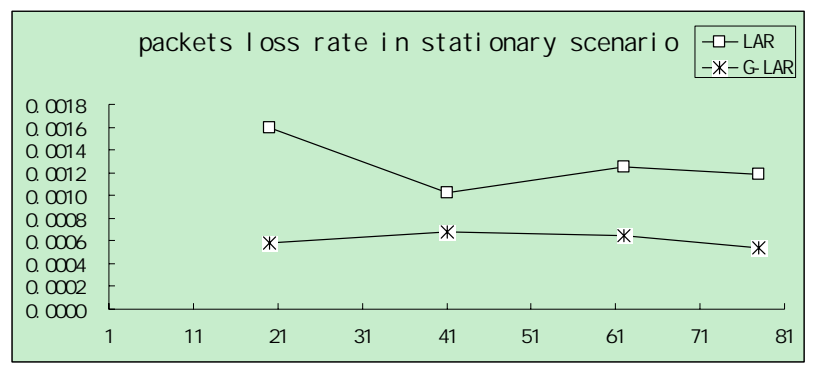

Fig. 11. Packets loss rate in stationary scenario

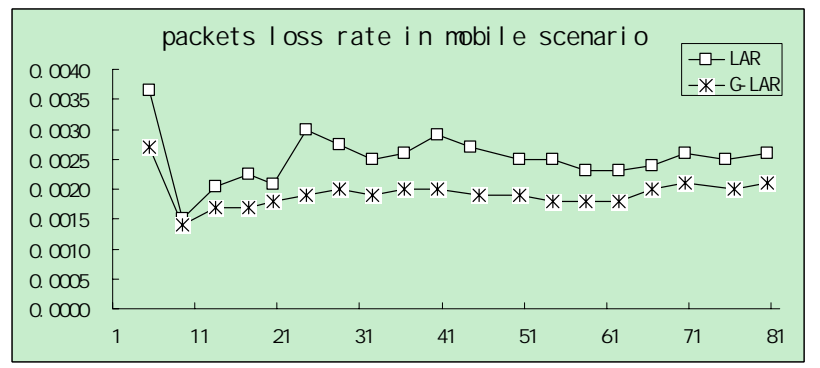

Fig. 12. Packets loss rate in mobile scenario

\section{Conclusion}

In this paper, we proposed the Grover location-aided routing (G-LAR) algorithm based on the idea of Grover search algorithm for MANET to reduce the overhead on the route-discovery. The key idea of the G-LAR algorithm is to selectively choose the appropriate nexthops in the request-zone for deliveries of control packets based on Grover search. Simulation results show that G-LAR algorithm lowers the overhead and reduces the computational complexity for routing effectively.

\section{Acknowledgements}

This work is supported by National Natural Science Foundation of China (60872020) and Zhejiang Provincial Natural Science Foundation of China (Y1101077).

\section{References}

1. H. Y. Yu, Mobile Ad Hoc Network (Posts \& Telecom Press, 2005), pp. 23-24.

2. M. A. Zayene and N. Tabbane, Performance evaluation of location-aided routing protocols in Ad hoc networks, Global Information Infrastructure Symposium, (2009) pp. $1-6$.

3. M. Bakhouya and N. Cottin, Performance evaluation of the location-based protocol DREAM for large mobile Ad Hoc networks, New Technologies, Mobility and Security (2008), pp. 1-6.

4. M. A. Zayene, N. Tabbane and R. Elidoudi, Performance evaluation of greedy perimeter stateless routing protocol in Ad Hoc networks, Proceedings of Computer Sciences and Convergence Information Technology (2009), pp. 907-912.

5. Z. Chen, K. Liu and J. Zhang, A novel location-based routing algorithm for mobile Ad Hoc network, Journal of Xidian University, 34(1) (2007), pp. 149-153.

6. L. K. Grover, A fast quantum mechanical algorithm for database search, Proceeding of the $28^{\text {th }}$ Annual ACM Symposium on Theory of Computing (1996), pp. 212-219.

7. Y. Cheng, C. H. Huang and W. M. Shi, Trusted dynamic source routing protocol. Proceeding of Wireless Communications, Networking and Mobile Computing (2007), pp. 1632-1636.

8. Q. H. Wang, C. Q. Gong and G. X. Wang, Locationaided routing protocol in mobile Ad Hoc network, Minimicro Systems26(11), (2005), pp. 1890-1892.

9. P. C. Li and S. Y. Li, An improved measure in Grover quantum searching algorithm, CAAI Transactions on Intelligent Systems,2(1), (2007), pp. 35-39.

10. L. Sun and W. B. Xu, Quantum search algorithm system and its applications, Computer Engineering and Applications(2006), pp.14-55.

11. M. A. Nielsen and I. L. Chuang, Quantum Computation and Quantum Information, (Higher Education Press, Beijing, 2003), pp. 230-231.

12. K. Arima, H. Miyajima, N. Shigei and M. Maeda, Some properties of quantum data search algorithm, Proceeding of The $23^{\text {rd }}$ Int. Technical Conf. on Circuits/ Systems, Computers and Communications (2008), pp. 1169-1172.

13. P. J. Salas and A. Gomez-Gonzalez, Robustness of quantum Grover algorithm against decoherence, AIP Conf. Proc (2007), pp. 261-262.

14. L. M. Meng, K. Zhou, X. Y. Shen and Z. J. Xu, Research on routing algorithm for mobile Ad Hoc networks based 
Hong Peng, et al

on Grover searching theory, Chinese Journal of Sensors and Actuators 23(2), (2010), pp. 251-255.

15. M. Zhang and C. T. Chang, OPNET and Network Simulation (Posts \& Telecom Press, Beijing, 2007), pp. 5-15. 